\title{
Fast and Accurate Sonar Trackers using Double Pulse Coding
}

\author{
Lindsay Kleeman \\ Intelligent Robotics Research Centre \\ Department of Electrical and Computer Systems Engineering, Monash University \\ Australia
}

\begin{abstract}
A new sonar target tracking system is presented that is capable of accurately tracking targets at measurement rates exceeding $10 \mathrm{~Hz}$. Two sonar trackers, each consisting of a transmitter and two receivers, are independently controlled to track sonar targets from bearing and range measurements. Bearing and range are accurately estimated using matched filters on two closely spaced receivers, with accuracy better than 0.1 degrees in still air conditions. Accuracy degrades with increasing air turbulence and temperature gradients, and bearing errors are shown experimentally to have significant autocorrelation at times of the order of seconds. The transmitter identity is coded using time separation of double pulses, thus allowing the two transmitters to operate simultaneously without spurious crosstalk readings. The ability to reliably reject interference is demonstrated experimentally. A simple computationally lean double pulse validation approach is analysed and experimentally tested with robot speeds up to 1 metre per second where Doppler shifting of the double pulse separation is an important factor.
\end{abstract}

\section{Introduction}

There are many sensing options available on mobile robots for application to localisation, map building and obstacle avoidance. Active sensing approaches such as laser range finders, stripe light ranging, infra-red sensors, short wave radar and sonar offer advantages in accuracy, robustness and simplicity compared to passive sensor systems such as stereo vision and passive infra-red sensing. A common problem with the active sensing approach is interference between different systems deployed concurrently. This paper addresses this problem in sonar sensing by reliably and simply rejecting interference between two or more sensors insonifying the same features simultaneously with minimal computational overhead and in one measurement. Other work on sonar interference has been published. Borenstein [1] has employed alternating firing patterns to statistically eliminate crosstalk problems, but requires more than one measurement cycle to reject interference. Pseudo random pulse sequences have been used in sonar [2], but incur a significant processing overhead and require long pulse sequences.

Despite the appearance of sonar systems capable of angle measurement and target classification [3, 4, 5, 6 7], there is still a perception that sonar sensing is unreliable and inaccurate in bearing [8,9], due mainly to inappropriate modelling that neglects fundamental wave physics (eg specularity and diffraction), crosstalk in sonar rings and poor sensor configurations. One poor sensor configuration is the popularly deployed Polaroid Ranging Module [10, 9] which has particularly poor bearing accuracy due to reliance on the angular discrimination of the transducer beamwidth. In fact one of the greatest assets of sonar is its bearing angle sensing accuracy and this will be shown in this paper. The other advantage of sonar sensing compared to shorter wavelength vision based systems is that the specularity of ultrasound in indoor environments naturally selects reliable map features such as smooth walls, corners and edges that are usually sparsely distributed in the environment. By using these natural beacons, accurate feature maps have been built while simultaneously localising to the features as the map is built [11].

Two new ultrasonic target trackers have been mounted on a mobile robot for a real time localisation and map building research project. This paper presents the first results from this work on the sonar trackers. The purpose of the sonar trackers is to quickly seek out and lock onto reliable sonar targets in the environment as the robot moves, similar to the sonar trackers in OxNav [3] and a 3D tracker [7]. However, the sonar trackers presented here produce more accurate bearing angle estimates and reliably reject interference from other sonar sources. The sonar tracker selects targets based on their sonar echo amplitude and pulse shape to ensure the targets are good candidates for localisation - these parameters were not available from the sonar trackers in the OxNav project.

The paper describes in Sections 2 and 3 the sonar tracker configuration and data acquisition hardware that transfers full receiver waveforms into processor memory in real time via the PCI bus. The data processing to extract arrival times and bearing angles is presented in section 4 . In order to reject interference, transmitter pulse coding is implemented. The coding approach and associated Doppler effects are analysed in Section 5 . Section 6 outlines the control of the sonar trackers. Experimental results on sonar interference and bearing accuracy are presented in Sections 7 and 8.

\section{Sonar Tracker Configuration}

The sonar tracker has a single transmitter and two separate receivers. Although it is possible to use the one transducer as a transmitter and receiver, a better signal to noise ratio is obtained by separating the two. Conventional wisdom suggests that greater bearing angle accuracy can be 
obtained by wider separation of the receivers [6]. Just the opposite approach is adopted here for several reasons:

- Arrival time jitter is highly correlated in space (and time) so that the difference in arrival times has relatively little jitter compared to absolute arrival time [4] for closely space receivers. This offsets the effect of a close receiver spacing.

- Correspondence of echoes on the two receivers is more reliable for closely spaced receivers.

- Highly accurate matched filter arrival time estimates [4] are employed so that accurate difference in arrival times can be obtained.

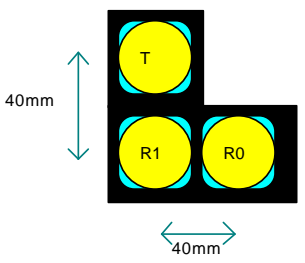

Figure 1 Placement of Polaroid 7000 series transducers as transmitter and receivers in the sonar tracker.

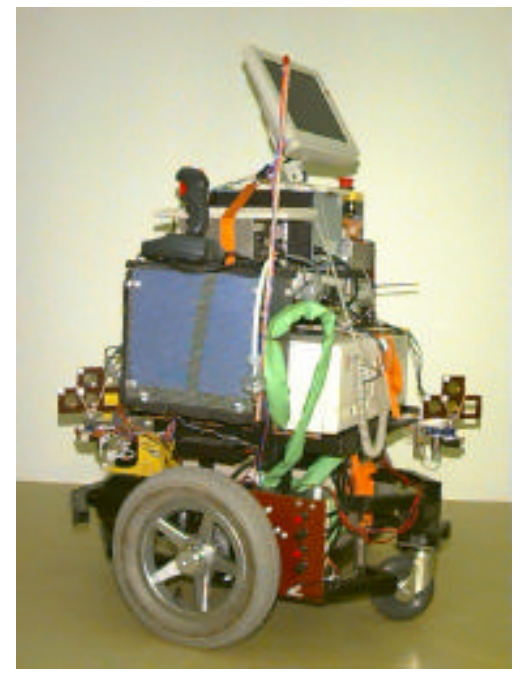

Figure 2 Mobile robot and positioning of front (left) and rear (right) sonar trackers.

The sonar tracker orientation is controlled by a DC motor and gearbox with optical encoder feedback from the pan angle to a MC1401 PID controller [12]. The encoder resolution is 0.18 degrees. The pan angle is driven at speeds of up to 360 degrees per second for rapid positioning.

Two sonar trackers are deployed on the robot at the front left and rear right as shown in Figure 2. This enables complete target "visibility" around the robot.

\section{Data Acquisition and Transmit Hardware}

In order to achieve accurate echo arrival time estimates using matched filtering, a sample rate of $1 \mathrm{MHz}$ is employed on each receiver. Each sample is digitised to 12 bits resolution and transferred to processor memory in real time via a PCI bus interface using DMA. A Pentium processor is then free to execute code whilst sonar data is transferred to memory. All four receiver channels are synchronised and share common control logic.

The analogue preprocessing circuitry employed is shown in Figure 3, and uses a low noise preamplifier AD797 and variable gain amplifier AD600 which are integrated circuits specially designed for a sonar front end. The variable gain amplifier prevents saturation for near targets and provides adequate gain for long range targets. This allows ranging from 0.07 to 8 metres. As echoes are received, the gain is varied by onboard RAM memory with $\mathrm{DAC}$ and low pass filtering, so that the gain profile can be adjusted in software. A 4th order low pass filter removes most receiver nois Gain Generator r anti-aliasing and thresholding purpos $\mathrm{RAM} / \mathrm{ADC}$

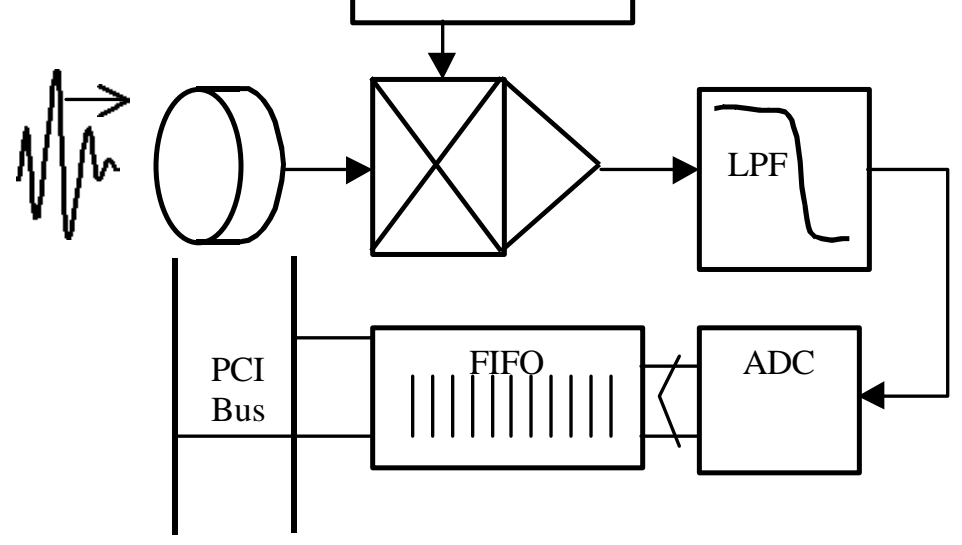

Figure 3 - Receiver interface and data acquisition circuitry for one receiver.

The transmitter excitation sequences are stored in a dedicated RAM with 1 bit coding for each transmitter sample, so that 300 Volts is represented by a 1 and 0 Volts by a 0 for each 0.5 microsecond interval. Thus the transmitted pulse shape is programmed in software. This is required for the double pulse coding scheme described in Section 5. Transmitted pulses are approximately 80 microseconds in duration (about $27 \mathrm{~mm}$ distance in air) and are sufficient energy to range out to typically 8 metres. Such short pulses allow fine target discrimination in range of the order of $15 \mathrm{~mm}$ separation (ie $30 \mathrm{~mm}$ distance of flight difference). 


\section{Sonar Data Processing}

The software to process the sonar data has been optimised for speed. Due to the high signal to noise ratio obtained by the careful design of the sonar front end electronics, the data processing has been significantly simplified. In particular, matched filtering [4] is performed only on sections of the echo signal deemed to be "pulses" by virtue of exceeding a threshold. The threshold varies with range and is set to 7 noise standard deviations computed online. Other sonar systems reported in the literature [6, 2] perform expensive matched filtering on the entire received waveform since pulses cannot be separated reliably from noise as can be done in this system. Examples of pulses extracted are shown in Figure 4. A pulse commences 30 samples before exceeding the noise threshold and ends 30 samples after falling below the threshold. A further process of "pulse splitting" is applied to separate nearly overlapping pulses by searching for a local maximum within a 60 sample sliding window.

The extraction of pulses from the received signals is performed as the echo signal arrives by using staggered DMA transfers from the receiver FIFO. Real time performance for pulse extraction has been obtained on a Pentium $120 \mathrm{MHz}$ computer running the real time multitasking operating system RTKernel [13].

Subsequently, the sonar pulses are processed to check double pulse validity as described in Section 5 and then matched filtering is performed on the acceptable pulses. The matched filter is a well known optimal arrival time estimator common in radar and is only briefly reviewed here. It consists of correlating the pulse with time shifted pulse templates and interpolating the best three correlations [4]. The templates are known pulses shapes for varying arrival angles and ranges. To speed up processing, the template is selected, not by finding the best correlation across a range of templates as in [4], but by choosing the best match between template and arrival pulse based on the zero crossing interval nearest to the pulse maximum. This zero crossing interval is used in [5] to estimate arrival angle with just one receiver but with larger angle errors than the current technique.

The processing time achieved for matched filtering is typically 20 milliseconds per receiver. The total measurement cycle time for one sonar tracker operating is typically $90 \mathrm{msec}$, and for two sonar trackers $140 \mathrm{msec}$ on a Pentium $120 \mathrm{MHz}$ processor.

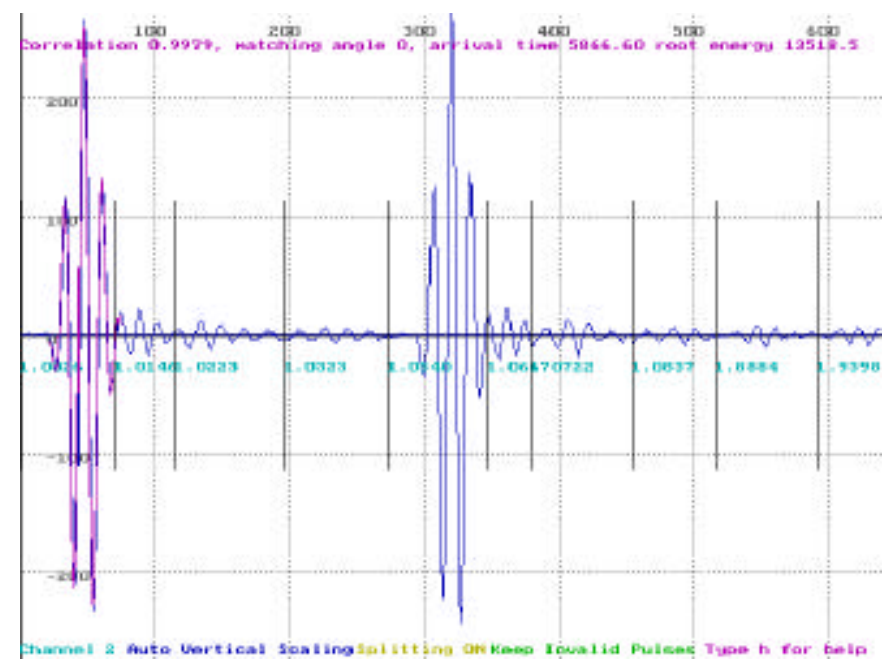

Figure 4 - Echo pulses after threshold extraction. An overlaid template appears on the left most pulse.

\section{Double Pulse Coding and Doppler Effects}

Interference between sonar trackers or with other robot ultrasonics sensors needs to be rejected for reliable operation. Sonar researchers $[2,6]$ have employed pseudo random and Barker pulse coding with durations of 1 to 2 milliseconds that requires the use of DSP systems to process the resulting echoes. The approach taken in this paper is simpler and consequently much faster to process. Each transmitter fires two precisely timed pulses with a separation dependent on the identity of the transmitter. The receivers can then identify arrivals by searching for the correctly matching second pulse. By subtracting one pulse (delayed appropriately) from another pulse in the time domain, very fast and sensitive detection can be achieved since both amplitude and arrival time need to match. Delays of less than $0.5 \mu \mathrm{sec}$ can be discriminated, making the possibility of false identification very remote. This sensitive detection has a drawback - the effect of robot velocity gives rise to a Doppler shift in the double pulse separation. The current implementation has been designed to tolerate robot speeds up to 1.3 metres per second.

After accounting for the transmitted time separation of pulses, the difference of two received pulses takes the form:

$$
\begin{aligned}
\operatorname{diff}(t) & =A p(t)-A(1+\delta) p(t+\Delta t)+n(t) \\
& =A[p(t)-p(t+\Delta t)-\delta p(t+\Delta t)]+n(t)
\end{aligned}
$$

where $p(t)$ is the pulse shape, $n(t)$ models the noise difference in the two received signals and $\delta$ and $\Delta t$ model the effects of amplitude and arrive time fluctuations respectively due to air disturbances and Doppler shifts when sensor or target relative motion occurs. For relative speeds much smaller than the speed of sound there is negligible compression or expansion of pulse shape $p(t)$. 
As can be observed in Figure 4 the pulses employed have limited bandwidth and can be decomposed into a sinusoidal component $\sin (2 \pi t / T)$ modulated by a slower varying amplitude $a(t)$. For small $\Delta t$ :

$$
\begin{aligned}
p(t)- & p(t+\Delta t) \cong \Delta t \frac{d p(t)}{d t}=\Delta t \frac{d(a(t) \sin (2 \pi t / T))}{d t} \\
& =\Delta t\left[a(t)(2 \pi / T) \cos (2 \pi t / T)+\frac{d a(t)}{d t} \sin (2 \pi t / T)\right] \\
& \cong(2 \pi \Delta t / T) a(t) \cos (2 \pi t / T)
\end{aligned}
$$

where the derivative of $a(t)$ is negligible compared to the derivative of the sinusoidal component. A simple measure, called $D$, of the difference is the maximum absolute difference normalised to the amplitude of the pulse. From equations (1) and (2):

$D=\max \left|\frac{\operatorname{diff}(t)}{A}\right| \cong \max \left\{\delta, \frac{2 \pi \Delta t}{T}\right\}+\frac{n\left(t_{\max }\right)}{A}$

where the $\boldsymbol{\delta}$ term maximum occurs when the derivative term is zero and vice versa. Due to the strong time correlation of amplitude fluctuations, $\delta$ is usually $\ll 0.2$ and can be ignored in comparison to the derivative term. The noise, if small, is only of consequence at the time of the maximum difference, $t_{\max }$.

We now consider the Doppler shift due to relative motion of the sensor and target. If a double pulse separation of $\tau$ is employed, a relative velocity $v$ results in a time shift of

$\Delta t=\frac{2 \tau v}{c}$

where $c$ is the speed of sound, and the 2 is due to both the transmitted double pulse and the received double pulse separations being effected by the motion. Shorter $\tau$ 's result in proportionally shorter time shifts and thus are more robust to relative motion. Values of $180 \mu \mathrm{s}$ and $200 \mu$ s have been chosen for the two transmitters. So that subsample pulse interpolation is not required when computing the delayed difference, $1 \mu$ s (ie the sample time) incremental shifts in the comparison are considered. Therefore the most $\Delta t$ can be is $0.5 \mu$ s which corresponds to a speed of $0.43 \mathrm{~ms}^{-1}$ from equation (4). For a measured period $T$ of $14 \mu$ s, equation (3) predicts a $D$ of 0.224 plus the signal to noise ratio term and this has been confirmed with real pulse data. In practice a threshold for acceptability for $D$ has been set at 0.25 and single $1 \mu \mathrm{s}$ shifts are tried in both directions. Thus values of $\Delta t$ up to $1.5 \mu \mathrm{s}$, which corresponds to $1.3 \mathrm{~ms}^{-1}$, should be tolerated. Experimental trials found that double pulses were accepted for speeds to $1.0 \mathrm{~ms}^{-1}$. Speeds above $1 \mathrm{~ms}^{-1}$ were not tested.
In summary, a set of pulses is tested in order of arrival as follows. All pulses are initially classified as "untagged", and each untagged pulse is tested for sufficient overlap with a successive "untagged" pulse before delayed subtraction is performed. Should the maximum absolute value of the difference divided by the pulse maximum (ie $D$ ) fall under 0.25 the first pulse is deemed "valid first" and the second pulse deemed "valid second" and these pulses are then excluded from further comparisons. If the $D$ is above 0.25 and under 0.5 a shift of plus or minus one sample (ie $1 \mu \mathrm{s}$ ) is applied and a new $D$ tested. The direction of the one sample shift is determined by examining the neighbouring samples at the point of maximum difference in the first $D$ computation.

\section{Sonar Tracker Control}

Each of the two sonar tracker pan angles is controlled independently whilst the firing of the transmitters is synchronised due to the hardware implementation. The sonar tracker has two simple modes:

(i) Target Searching - higher level commands define the search angle over which the sonar tracker scans. The tracker pans to the starting angle at $350 \mathrm{deg} / \mathrm{sec}$, and then pans to the final angle at $90 \mathrm{deg} / \mathrm{sec}$ whilst firing continuously. The largest amplitude echo that matches a template with a correlation coefficient higher than 0.85 is selected. This procedure aims to select, within the bounds set by the search angle range, an uncluttered reliable target for tracking.

(ii) Target Locking - from the initial pan angle supplied from target searching, the sonar continuously fires and from echoes within a time of flight attention window finds the bearing angle to the largest amplitude target. This angle is used as an error signal to control the pan angle so that the tracker points to the target. The time of flight attention window is set to a fixed time before and after the previous sonar target time of flight and enables range discrimination should multiple targets produce echoes. It also speeds up sonar data processing. A "no lock" situation arises when no targets are detected within the attention window. After a fixed number of consecutive "no locks", target locking is abandoned and a new search commenced. This allows for occasional loss of tracking due to, for example, people walking past the target. Changes in robot odometry orientation are employed to assist target locking.

\section{Interference Experiments}

Although the sonar trackers have been deliberately positioned on the robot to minimise crosstalk problems, it is still possible for both trackers to track the same target. To illustrate the interference rejection capability of the sonar trackers, an experiment has been devised to track a plane target at a range of 3.5 metres with both sonar 
trackers whilst the robot slowly rotates as shown in Figure 5.

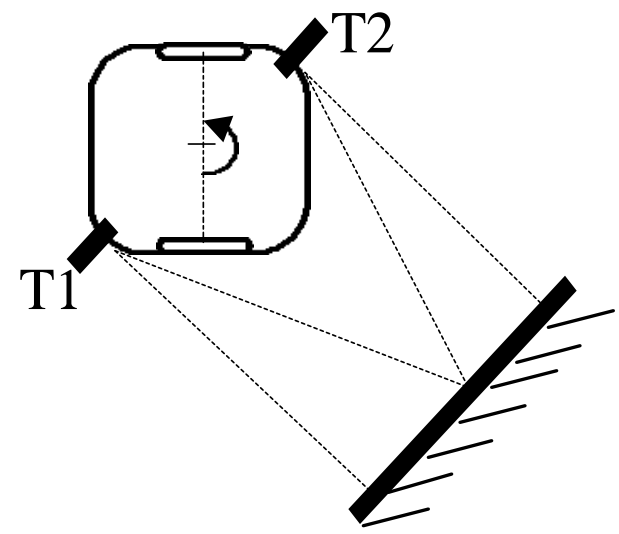

Figure 5 - Experimental arrangement for studying interference.

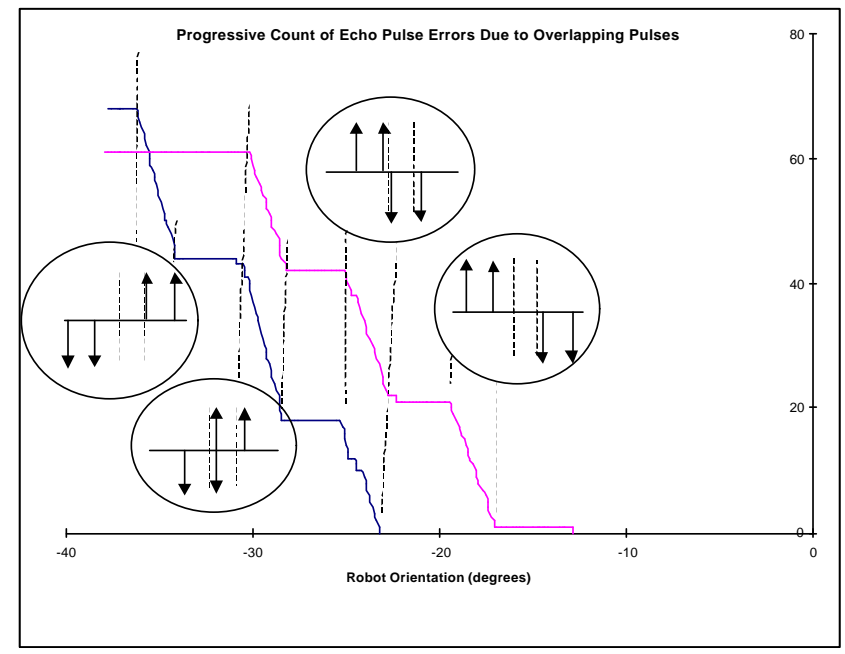

Sonar Bearing Estimates of the Common Plane

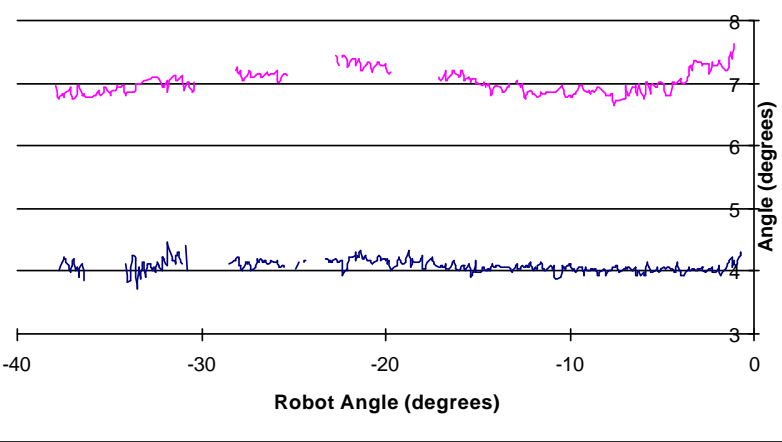

Figure 6 - Progressive count of tracker failures due to overlapping pulses.

Figure 7 - Tracker target bearing estimates (offset for clarity). Gaps result from overlapping pulses.

As the robot rotates, each sonar tracker receives pulses from the other tracker that gradually shift past the pulses of interest. The crosstalk pulses are rejected by the delayed pulse subtraction verification technique described in Section 5. However when two pulses overlap, the arrival time cannot be determined by matched filtering and the target tracker reports a "no lock". The cumulative total of the "no locks" (where no range and bearing are reported) are plotted against the robot angle in Figure 6 as the robot angle decreases from right to left. Overlap conditions are represented in the timing diagrams in the circular reliefs up arrows represent front tracker pulses, down arrows represent rear pulses and dashed lines represent crosstalk pulses. For example, from -18 to -20 degrees the rear tracker first pulse overlaps with the crosstalk second pulse from the front tracker. The sonar trackers are 0.33 metres from the robot centre of rotation and the 2 degree overlap intervals correspond to $1 \mathrm{~cm}$ of movement of each tracker towards and away from the plane.

The tracker target bearing estimates combined with odometry orientation are plotted in Figure 7 from the same experiment. An offset of 3 degrees between the two angles is of no significance and allows clearer presentation. The gaps in the data correspond to the pulse overlap conditions. It should be stressed here that overlapping pulses are usually a rare event and the results presented here are intended to illustrate the worst case scenario.

\section{Bearing Accuracy Results}

This section presents bearing results obtained from the sonar sensor on the target tracker across the full available beamwidth of the sensor. Note that the beamwidth available depends on range, target reflectivity and ambient air conditions. The angle to a plane at a range of 2.75 metres was measured with 200 readings at each 1 degree increment. The mean plus and minus two standard deviations of the angles are plotted in Figure 8. Although the discrepancies between different measurements are small, they are statistically significant. One obvious source of error is the optical encoder resolution of 0.18 degrees. Another source of error is air turbulence combined with temperature gradients. Sound propagation is refracted by speed of sound gradients resulting from temperature gradients. By observing the angle output in real time, significant variations up to 0.5 degrees lasting several seconds have been observed as people walk past the tracker (but not blocking the echoes). Placing a hot object, such as a cup of coffee under the air column of propagation results in large random angle and amplitude fluctuations.

In order to study this further, experiments were conducted in a laboratory where cold air draughts mix with the warmer air in the laboratory. The autocorrelation of the angle errors is plotted in Figure 9. A cyclic variation of errors of approximately 5 seconds is apparent in the angle errors. It is clear that the error spectrum is coloured and any future sensor error models should include non-white noise assumptions. 


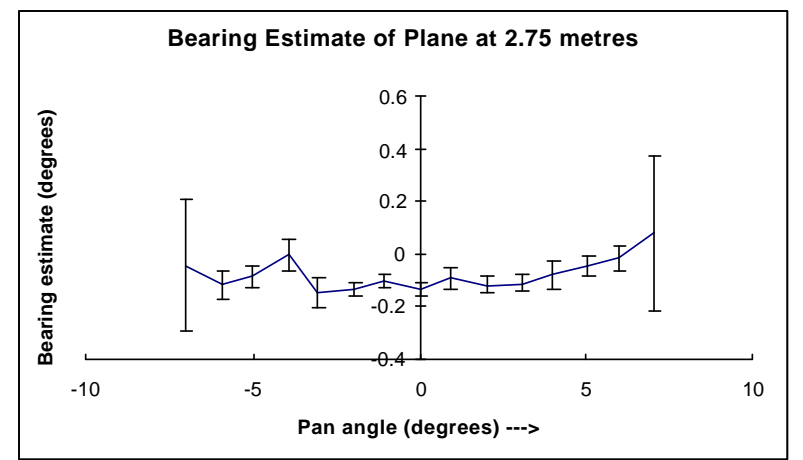

Figure 8 - Bearing angle measurements to a $2.75 \mathrm{~m}$ range plane. Mean, plus and minus 2 standard deviation errors bars are shown.

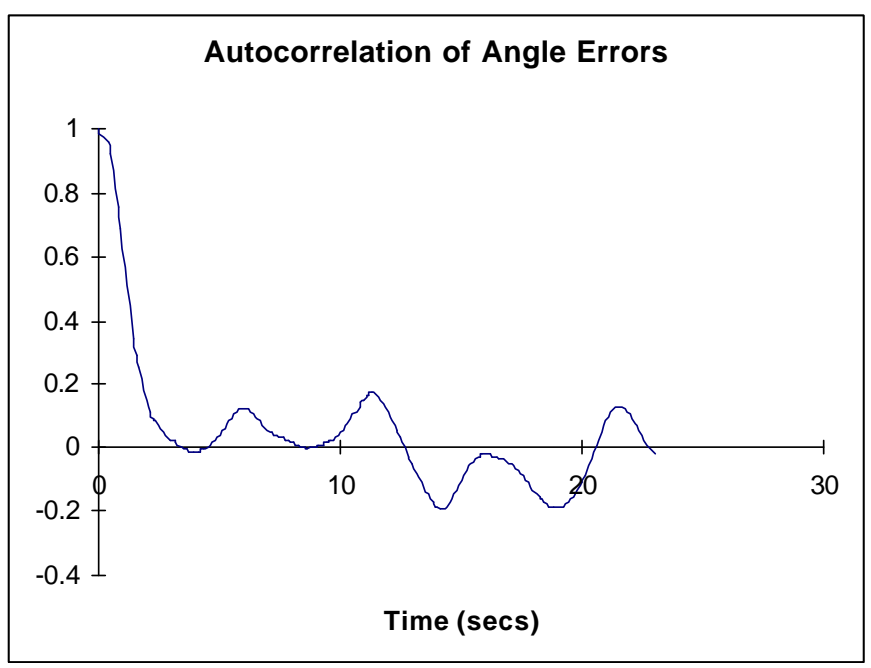

Figure 9 - Autocorrelation of 1000 bearing measurements - sampled at $10 \mathrm{Hertz}$ from a plane at $1.1 \mathrm{~m}$ in draughty conditions (stand. dev. of $0.067 \mathrm{deg}$ ).

\section{Conclusions and Future Work}

A new sonar tracking system has been presented that rejects interference from other sonar systems using a simple double pulse coding scheme that results in efficient processing. The system operates sufficiently rapidly for application to real time map building and localisation problems. The sonar sensor range and bearing errors are so low that ambient air conditions are the main contributing factor to errors. The effects of air turbulence and temperature mixing on sonar bearing estimation has been highlighted in this paper as an area requiring future attention by sonar researchers. Future work on real time directed sensing for map building and localisation will be pursued with the robot and sonar trackers.

\section{Acknowledgments}

This work is funded by a large Australian Research Council grant. The professional engineering work of Greg Curmi and Steve Armstrong are gratefully acknowledged.

\section{References}

[1] J Borenstein and Y Koren, "Error eliminating rapid ultrasonic firing for mobile robot obstacle avoidance", IEEE Trans. Robotics and Automation, Vol 11, No. 1 pp 132-138, 1995.

[2] K. Jorg and M. Berg, "Mobile robot sonar sensing with pseudo-random codes", Proceedings 1998 IEEE Conference on Robotics \& Automation, Leuven, BelgiumMay 1998, pp 2807-2812.

[3] A Stevens, M Stevens and $H$ Durrant-Whyte, "'OxNav': Reliable autonomous navigation", IEEE International Conference on Robotics and Automation 1995, pp 2607-2612.

[4] L. Kleeman and R. Kuc, "Mobile robot sonar for target localization and classification", International Journal of Robotics Research, Volume 14, Number 4, August 1995, pp 295-318.

[5] T Yata, L. Kleeman and S Yuta, "Fast bearing measurement by a single ultrasonic transducer and its application to mobile robot wall following", Proc. IEEE International Conference on Robotics and Automation 1998, Leuven, Belgium May 1998, pp. 1590-1596.

[6] H. Peremans, K. Audenaert, and J. M. V. Camperhout, "A high-resolution sensor based on tri-aural perception," IEEE Transactions on Robotics and Automation, vol. 9, pp. 36-48, 1993.

[7] R. Kuc, "Three dimensional tracking using qualitative sonar," Robotics and Autonomous Systems, vol. 11, pp. 213-219, 1993.

[8] J. Budenske and M. Gini, "Why is it so difficult for a robot to pass through a doorway using ultrasonic sensors?," IEEE Conference on Robotics and Automation, San Diego CA, 1994 pp. 3124-3129.

[9] T. Tsubouchi, "Nowadays trends in map generation for mobile robotics", Proceedings IROS'96, Osaka Japan 1996, pp 828-833.

[10] D. Lee, The map-building and exploration strategies of simple sonar-equipped mobile robot, Cambridge University Press 1996.

[11] K S Chong and L. Kleeman "Large Scale Sonarray Mapping using Multiple Connected Local Maps", International Conference on Field and Service Robotics, ANU December 8-10, 1997, pp. 538-545.

[12] Performance Motion Devices, "Advanced multi-axis motion control chipset MC1401", Concord MA 1995.

[13] On Time Informatik GMBH, RTKernel 4.5 Real-time multitasking kernel for C/C++ User's Manual, 1995. 